\title{
An investigation using high-precision CCD laser displacement sensor to measure body surface motion induced by heartbeat
}

\author{
Satoshi Suzuki ${ }^{1}$, Takemi Matsui ${ }^{2}$, Takafumi Asao ${ }^{1}$, Kentaro Kotani ${ }^{1}$ \\ ${ }^{1}$ Department of Mechanical Engineering, Faculty of Engineering Science, Kansai University, Osaka, Japan \\ ${ }^{2}$ Department of Management Systems Engineering, Faculty of System Design, Tokyo Metropolitan University, Tokyo, Japan \\ Email: ssuzuki@kansai-u.ac.jp
}

Received 22 August 2012; revised 23 September 2012; accepted 5 October 2012

\begin{abstract}
The aim of this study was to measure the motion of the body surface induced by heartbeat by using a high-precision laser sensor in order to provide a reference for other research on medical remote sensing techniques that are unconstrained and non-contact. Body surface motions at some points on the chest and on the dorsal region were measured for eight men with a laser displacement sensor. For comparison, an electrocardiograph was also used to monitor the heartbeat. As a result, we confirmed cyclic motion of about $0.1 \mathrm{~mm}$ in amplitude everywhere on the body surface, not only from the front of the body but also from the back and the neck. In contrast, which part on the body was most suitable for measurement had to be taken into account because a difference in motion amplitudes was observed in different parts on the body. The amplitude of the motions also depended on the conditions of the body surface as well as underneath the skin. These results show the possibility of acquiring information about heartbeats from anywhere on the body by using an unconscious sensing technique, and the potential for the technique to monitor the condition of the body and personal physical characteristics.
\end{abstract}

Keywords: Unconstrained Sensing; Heart Rate; Body Surface; Motion

\section{INTRODUCTION}

Recently, to avoid the restrictions caused by the presence of lead wires and to remove the burden of attaching electrodes, medical remote sensing techniques that are nonrestrictive, non-invasive and non-contact for monitoring vital signs have been actively researched and developed (here, we refer to such techniques as "unconscious sens- ing”). Examples include sensors using a strain gauge [1], pressure sensors [2], or film of the piezoelectric polymer polyvinylidene fluoride (PVDF) [3]. In particular, the PVDF-based sensor can non-restrictively and non-invasively measure heartbeat and respiration on a person's dorsal body surface. In the current study, a method for completely non-contact vital signs monitoring using microwave radar is proposed. The method was originally developed to search for survivors trapped beneath earthquake rubble $[4,5]$. Microwave radar has the following characteristics: 1) the microwaves can be transmitted through most objects, except metals and water and 2) movement of an object can be detected from distance without actually needing to touch it. By applying this system to the monitoring of human vital signs, motion of the body surface can be observed from a distance without removing any clothing. For example, respiratory rates of examinees in bed were monitored through bedding by using a ceiling-mounted microwave radar [6]. The purpose of the current study is to develop a system not only to reduce the burdens on the elderly which are created by attaching electrodes to the body but also to reduce the burdens on nursing caregivers having to confirm the safety of elderly patients with frequent overnight visits. The microwave radar techniques used in this research have already been applied to monitoring the vital signs of a hibernating bear for more than three consecutive months in a zoological garden [7]. In another trial, a 24$\mathrm{GHz}$ compact microwave radar attached to the rear surface of a chair could monitor not only heart rate but also heart rate variability (HRV) [8].

These unconscious sensing techniques are aimed at detecting motion at extremely minute scales on the body's surface caused by cardiac motion. An important contribution to this subject area has been made by Lin, Droitcour et al. $[9,10]$ who have developed mathematical theory on non-contact sensing techniques and have constructed mathematical models under the assumption of 
monitoring body surface motions.

However, motions on the body surface induced by heartbeat are so small that they are barely apparent. As a consequence, the problem in previous research was the extrinsic noise cancellation and the removal of artefacts resulting from respiratory activity and motions caused by arm movement [11]. Nevertheless, none of these studies addressed the question of how many motions of different amplitude appear on the body surface. There are specific body parts that are generally considered to have the characteristics necessary for monitoring by unconscious sensing, owing to there being differences between the conditions on and under the skin for each part of the body.

Therefore, for use as a reference base for unconscious sensing research, the aim of the current study was to measure motions on the body surface induced by heartbeat by using a high-precision charge-coupled device (CCD) laser displacement sensor and to confirm the magnitude of these movements. Through observation of the motion on the surface of the chest wall and dorsal region, the applicability of the unconscious measurement method is discussed.

\section{METHODS}

\subsection{High-Precision CCD Laser Displacement Sensor Specifications}

To measure motion on the body surface, an industrial high-precision CCD laser displacement sensor (LK-G35; Keyence Co., Tokyo) was chosen. This red CCD laser has a $650 \mathrm{~nm}$ wavelength, a measurement resolution of $0.05 \mu \mathrm{m}$ and an effective measuring range of $\pm 5 \mathrm{~mm}$; its maximum sampling frequency is $50 \mathrm{kHz}$ and its maximum power output is $4.8 \mathrm{~mW}$. This type of laser displacement sensor is frequently used in measuring the thickness and evenness of a vehicle's paint finish, and in quality control to find scuffs of extremely minute scales on compact discs and hard disks.

If the laser beam from the laser displacement sensor is irradiated directly onto a person's skin, the beam, because of its low power, will not penetrate into the body and will not cause skin problems. However, to ensure maximum safety, we placed a piece of white vinyl stretch tape on the body surface and each participant wore laser protection goggles with an optical density of 3 .

\subsection{Measurement Positions and Participants}

The target positions V2, V3 and V5 (see Figure 1) for measurement of body surface motions, were decided from referral to a 12-lead electrocardiogram. Additionally, the left-side carotid artery was monitored (CA in Figure 1), because it is a representative artery that is

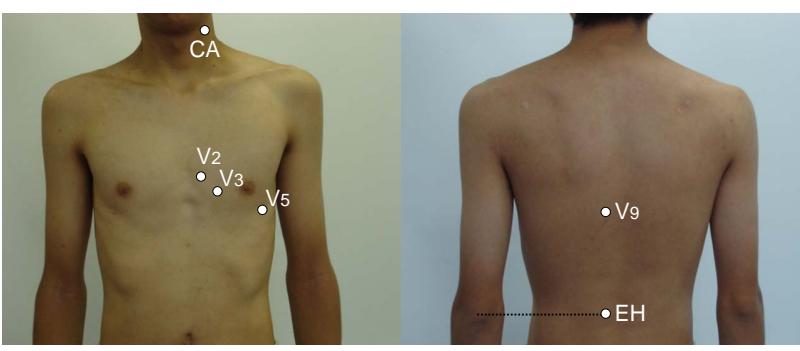

(a)

(b)

Figure 1. Measurement points. V2, V3 and V5 were decided from referred to 12-lead electrocardiogram. Additionally, the carotid artery in left side was also monitored (CA). Measurement positions on dorsal region V9 were chosen referred to 18-lead electrocardiogram and the elbow height $(\mathrm{EH})$ in lumber area was measured with reference to the industrial standard. (a) Front; (b) Back.

positioned close to the skin's surface. Conversely, the measurement position on the dorsal region, V9, was chosen as it referred to a 12-lead electrocardiogram [12], and to an earlier study by the authors that monitored the same position [8]. Furthermore, researchers [13,14] have attempted to detect heartbeat from the lumbar area; therefore, the motion on the elbow height (EH on Figure 1) in the lumber area was measured in accordance with industrial standard [15]. This position EH was stirred about $20 \mathrm{~mm}$ from midline to left side considered in the backbone.

Body surface motion was measured for eight men (mean age: $21.88 \pm 1.13$ years; range: 21 - 24 years). Additionally, to observe the relationship between the measurements and the personal physical characteristics of each participant, their subcutaneous fat thicknesses were found in advance (see Table 1). The subcutaneous fat around each measurement position was measured three times with skinfold callipers (MD-550; Yamasa Tolei CO. LTD., Tokyo) in reference to a previous study [16] and the average was calculated.

\subsection{Investigation Procedure}

To conduct the measurements, a participant, naked from the waist up, lay down on a urethane foam mattress and the laser displacement sensor was set on a steady aluminium frame above the body. The laser sensor was positioned $3 \mathrm{~cm}$ from the body surface and the movement measured at each target position (Figure 2). Additionally, to take measurements on the dorsal region, participants lay face down on the mattress. To remove any motion induced by respiration, we instructed each participant to hold a breath for $30 \mathrm{~s}$. For comparison, we also monitored an electrocardiograph (ECG) connected by precordial leads (WEB-5500; Nihon Koden Co., Tokyo) and collected information with a sampling frequency of 100 Hz. The output signal data from the laser distance sensor 
Table 1. Individual physical characteristics of eight volunteers.

\begin{tabular}{|c|c|c|c|c|c|c|c|c|c|c|c|c|}
\hline \multirow{2}{*}{ Subject } & \multirow{2}{*}{ Age } & \multicolumn{2}{|c|}{ Body dimension } & \multirow{2}{*}{$\begin{array}{c}\text { Body mass } \\
\text { index }\end{array}$} & \multicolumn{2}{|c|}{$\begin{array}{l}\text { Average of blood } \\
\text { pressure }(\mathrm{mmHg})\end{array}$} & \multicolumn{6}{|c|}{ Average of subcutaneous fat (mm) } \\
\hline & & $\begin{array}{l}\text { Height } \\
\text { (cm) }\end{array}$ & $\begin{array}{l}\text { Weight } \\
(\mathrm{kg})\end{array}$ & & Systolic & Diastolic & $\mathrm{V} 2$ & V3 & V5 & V9 & $\mathrm{EH}$ & Neck \\
\hline$S_{1}$ & 24 & 172 & 52 & 17.58 & 122.67 & 65.00 & 4.00 & 5.33 & 4.67 & 6.33 & 7.33 & 2.67 \\
\hline$S_{2}$ & 21 & 171 & 55 & 18.81 & 125.67 & 72.00 & 3.00 & 4.00 & 4.00 & 8.00 & 5.67 & 2.33 \\
\hline$S_{3}$ & 21 & 166 & 54 & 19.60 & 120.67 & 64.67 & 4.33 & 5.33 & 6.00 & 8.00 & 10.33 & 3.33 \\
\hline$S_{4}$ & 21 & 175 & 61 & 19.92 & 122.00 & 64.00 & 4.33 & 5.33 & 6.33 & 6.33 & 6.67 & 3.33 \\
\hline$S_{5}$ & 22 & 172 & 65 & 21.97 & 120.00 & 68.33 & 9.00 & 11.67 & 12.67 & 12.67 & 14.00 & 3.00 \\
\hline$S_{6}$ & 21 & 168 & 57 & 20.20 & 124.67 & 65.67 & 4.67 & 4.33 & 6.33 & 8.33 & 7.00 & 2.67 \\
\hline$S_{7}$ & 23 & 179 & 75 & 23.41 & 135.33 & 72.33 & 5.00 & 9.67 & 11.00 & 12.67 & 12.33 & 1.67 \\
\hline$S_{8}$ & 22 & 185 & 72 & 21.04 & 134.00 & 71.67 & 4.67 & 7.00 & 6.33 & 9.00 & 9.00 & 4.33 \\
\hline Average & 21.88 & 173.50 & 61.38 & 20.31 & 125.63 & 67.96 & 4.88 & 6.58 & 7.17 & 8.92 & 9.04 & 2.92 \\
\hline$S D$ & 1.13 & 6.12 & 8.57 & 1.82 & 8.40 & 4.73 & 1.78 & 2.73 & 2.97 & 2.41 & 2.88 & 0.97 \\
\hline
\end{tabular}

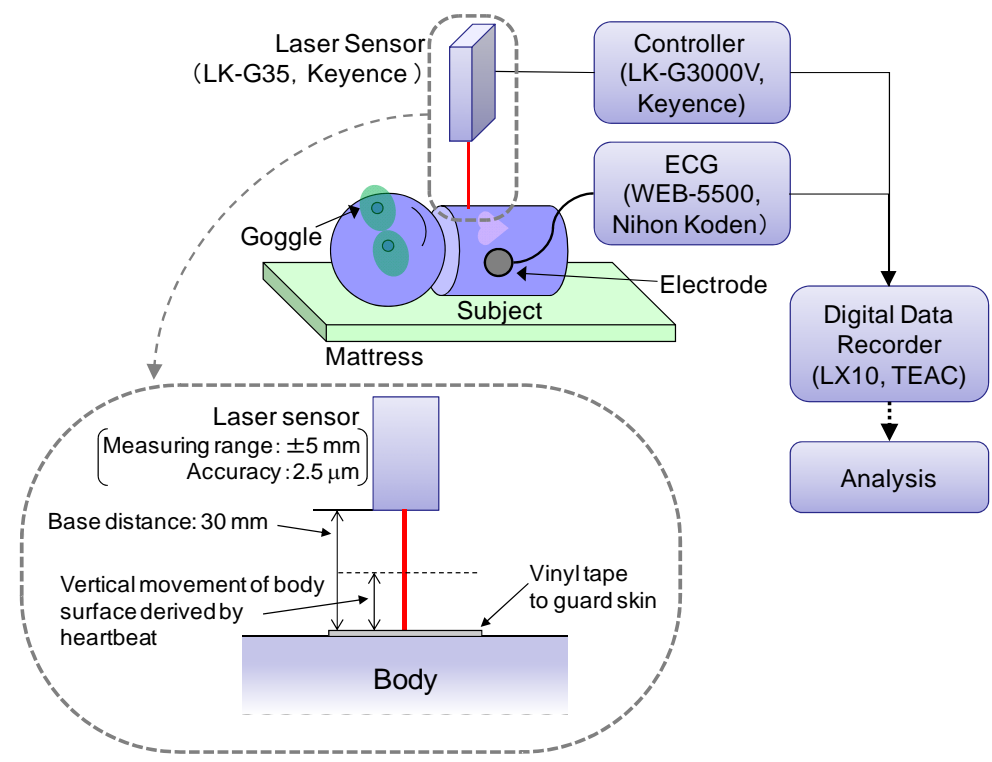

Figure 2. Schematic diagram of the experimental setup. A high precision CCD-laser displacement sensor was used to measure the motion on the surface of the body, and an ECG was also used as a reference, providing direct measurements with ECG.

were stored in synchronization with the ECG data in a digital recorder (LX-10; TEAC Co., Tokyo).

\section{RESULTS}

\subsection{Motions on the Body Surface}

Figure 3 shows $10 \mathrm{~s}$ of sample data monitored for subject S1, extracted from the total of $30 \mathrm{~s}$ of data. Figure 3(a) shows the output signal from the ECG and Figure 3(b) shows the output signal acquired by the high-precision CCD laser displacement sensor. It can be seen from this figure that the cyclic oscillation in the data acquired by the laser displacement sensor is synchronized with the R-waves from the ECG. Although some of the sensor data included baseline drift resulting from respiratory motion, this synchronous cyclic oscillation with the Rwaves of the ECG was clearly observed in all of the participants. Additionally, this cyclic oscillation has several peaks, similar to the pulse wave. This wave shape was confirmed at all measurement positions for all participants, despite the slight differences in the form of the motion on the body surface. Likewise, some previous 


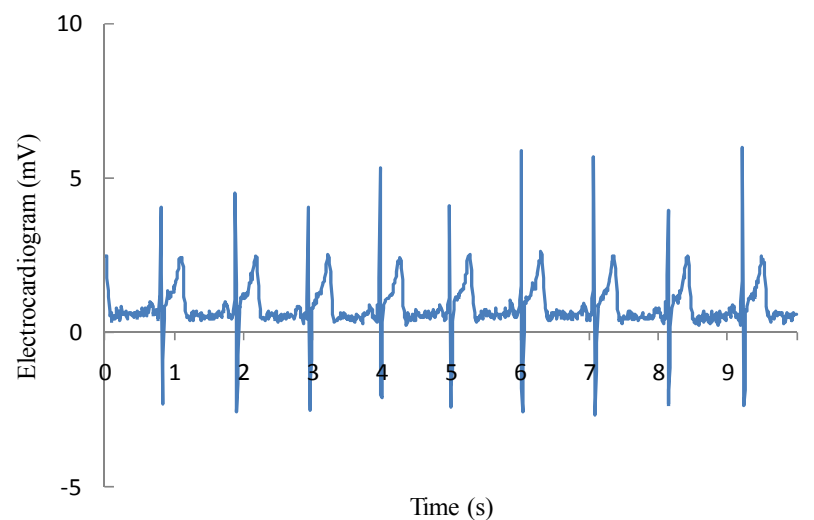

(a)

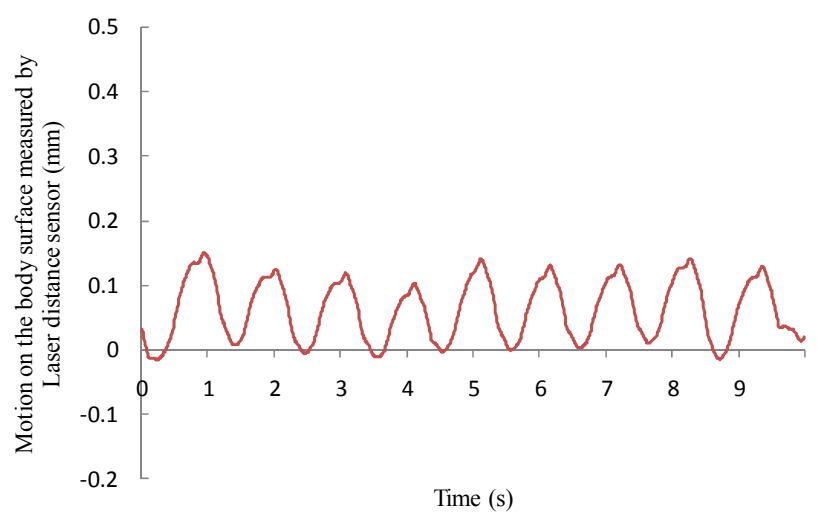

(b)

Figure 3. Sample data of subject S1 monitored for 10 seconds, extracted from the total 30 seconds of data; (a) shows the output signal from the ECG, (b) shows the output signal acquired by high precision CCD-laser displacement sensor. The cyclic oscillation synchronized with R-waves of ECG is showed in the data acquired by the laser displacement sensor. (a) Output signal of ECG; (b) Output signal of laser displacement sensor.

studies have reported that the shape of oscillations acquired by microwave radar when monitoring body surface motion have two or more peaks per cycle [8-10]. In a similar way to the pulse wave, these multiple peaks are thought to be due to changes of the arterial blood pressure.

\subsection{Difference between Measurement Positions}

Figure 4 shows the average amplitudes of the motions on the body surface at each measurement point. While there are differences between the amplitudes at each measurement position, cyclic oscillations are found at all positions. Especially, the amplitudes for the carotid artery are naturally large, because motion is measured directly on the artery. In contrast, the motions on the front of the body have amplitudes of about $0.1-0.2 \mathrm{~mm}$, and those on the back are about $0.1 \mathrm{~mm}$ high. It should be noted that the dorsal region does also move, despite this movement being slightly smaller than that for the front.

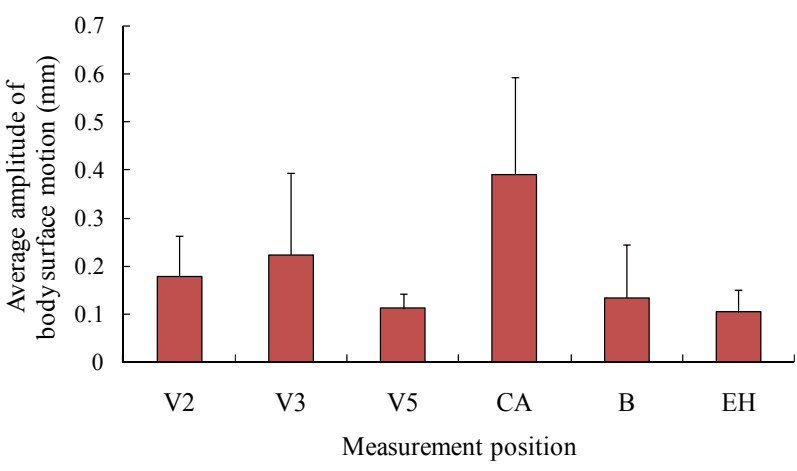

Figure 4. The results of the motion on the body surface. In order to compare between each measurement point of all subject. While there are some differences in amplitudes at each measurement position, the motions on the body surface are confirmed at all of measurement positions.

As a consequence, the dorsal region cannot be concluded to be inadequate for measuring vital signs. The difficultties in measuring vital signs from a person's back has been pointed out and discussed, however, heartbeat information can be extracted through unconscious sensing techniques, because the surface of the dorsal regions moves.

\subsection{Relation between Body Surface Motion and Subcutaneous Fat Thickness}

Figure 5 shows the relationship between body surface motions and subcutaneous fat thickness measured by skinfold callipers for all positions on all participants. A tendency for the amplitude of the body surface motion to decrease with an increase in subcutaneous fat thickness is clearly observed from Figure 5. Although the correlation coefficient by curve-fitting with an exponential function is not high $(r=0.63)$, the decreasing trend is confirmed and could be considered as a measure of the condition of the body surface.

\section{SUMMARY AND DISCUSSION}

We have investigated body surface motion induced by heartbeat by using a high-precision CCD laser displacement sensor. As a result, we showed that cyclic motion exist at all measured points on the body surface, not only for the front of the body but also for the back and the neck. Although the amplitudes of these motions were different for different parts of the body, the results indicate the possibility of acquiring heartbeat information from anywhere on the body in a straightforward manner by using unconscious sensing techniques.

Conversely, because difference amplitudes were observed for different parts on the body, the most suitable part on the body for measurement must be considered, taking into account the capability and operation of the 


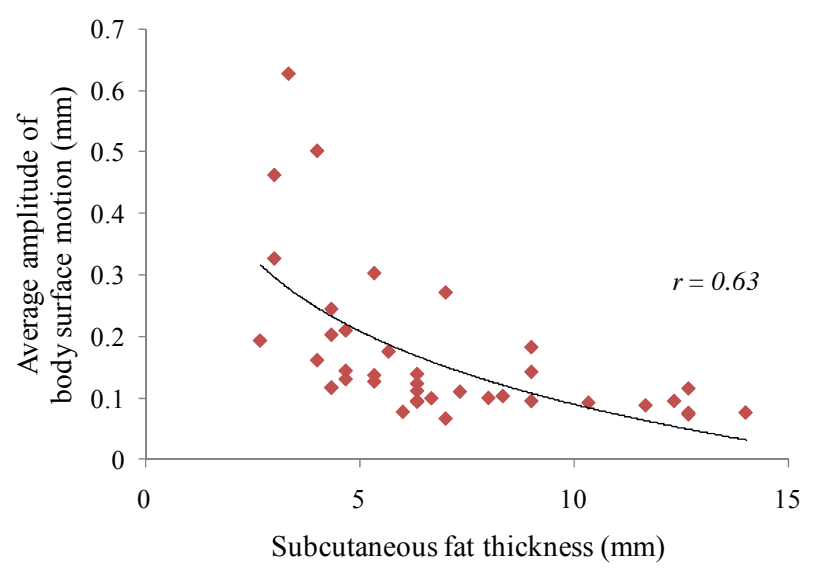

Figure 5. A result of a relationship between the motion on the body surface and subcutaneous fat thickness. A tendency to degrease the amplitude of the motion on the body surface in case of increase the subcutaneous fat thickness is observed.

sensors. We chose eight samples made up of people who have physiologically similar characteristics. However, as a result, it is important that this paper indicates the differences that do appear in such a homogenous sample. Namely, in the case of extracting information from the body surface motion, not only the target measurements but individuals' physical characteristics must be considered. It is presume that the results varying by age, gender, and other factors. Additionally, results depend on the hardness of the skin and the presence of burn injuries or skin diseases. The results of this report served a purpose by pointing out the importance of discussing such issues from the viewpoint of physiology for research about on unconscious sensing techniques. The investigations of the influences of other factors remain a topic for future research and discussion.

On the other hand, the meaning of the waveform of signals is also important. Waveforms of signals acquired by the high-precision CCD laser displacement sensor are unstable and also differ by subject. This character has been seen in previous research about unconscious sensing techniques $[9,10,13,14]$. Additionally, the waveforms in both this report and previous research are not simple waveforms like the sine wave. While differences are observed in the number of peaks and shoulders in different studies, some peaks and shoulders have been confirmed to appear in each cycle in most research [17-19]. This means some physiological information is included in every cycle of such signals as ECG signals and pulse waves. The pulse wave is acquired, for example, from the radial, brachial, and carotid arteries in upper body, and the femoral artery and posterior tibial artery in lower body. The shape of a pulse wave differs according to the measurement point [20]. A pulse wave at the carotid artery has typically 6 wave features, preejection waves, anacrotic shoulder, percussion wave, tidal wave, incisura and dicrotic notch. Moreover, in the pulse wave acquired at other points, some wave features do not appear clearly [20].

Additionally, the main peak of the pulse wave, called the percussion wave, occurs after the R-wave of the ECG and also occurs between the S-wave and the T-wave of the ECG. The main peaks of the output signals from the high-precision CCD laser displacement sensor at all measurement points appear just after the occurrence of the R-wave of the ECG. The output signals from the high-precision CCD laser displacement sensor have similar characteristics to a pulse wave. It is thought that the motion on the body surface includes information similar to a pulse wave, because the motion on the body surface is caused by the vibration induced by the blood flow in a peripheral vessel.

In general, most research on unconscious sensing techniques refers to ECG. Moreover, most non-contact sensing research generally considers only the relationship between the occurrence time of the R-wave of the ECG and the wave peak, and the other information included in the signals is not discussed. The reason for this seems to be that the waveforms of the signals acquired by unconscious sensing techniques are unstable and also differ by subject. However, it is seems that ECG is not sufficient for discussion of waveforms and the information included in waveforms, and pulse waves instead are appropriate as a reference for researches about on unconscious sensing techniques.

The pulse wave velocity is occasionally used for diagnosis of cardiovascular disease [21]. In the future, cardiovascular disease potentially could be diagnosed without directly touching the patient through detailed investigation of the relationship between the pulse wave and the output data acquired by a non-invasive sensing technique. If the cardiovascular information in the output data can be clearly understood, unconscious sensing techniques would be useful not only for patients who cannot be touched - for example, those with severe burn injuries and serious lacerations - but also for patients who have problems with cardiac functions.

\section{REFERENCES}

[1] Ciaccio, E.J., Hiatt, M., Hegyi, T. and Drzewiecki, G.M. (2007) Measurement and monitoring of electrocardiogram belt tension in premature infants for assessment of respiratory function. Biomedical Engineering Online, 6, $1-11$.

[2] Jacobs, J., Embree, P., Glei, M., Christensen, S. and Sullivan, P. (2004) Characterization of a novel heart and respiratory rate sensor. Conference Proceedings IEEE Engineering in Medicine and Biology Society, San Francisco, 1-5 September 2004, 2223-2226.

[3] Wang, F., Tanaka, M. and Chonan, S. (2006) Develop- 
ment of a wearable mental stress evaluation system using PVDF film sensor. Journal of Advanced Science, 18, 170173. doi: $10.2978 /$ jsas. 18.170

[4] Chen, K.-M., Huang, Y., Zhang, J.P. and Norman, A. (2000) Microwave life-detection systems for searching human subjects under earthquake rubble or behind barrier. IEEE Transactions on Biomedical Engineering, 47, 105-114. doi:10.1109/10.817625

[5] Chen, K.-M., Misra, D., Wang, H., Chuang, H.-R. and Postow, E. (1986) An X-band microwave life-detection system. IEEE Transactions on Biomedical Engineering, 33, 697-702. doi:10.1109/TBME.1986.325760

[6] Uenoyama, M., Matsui, T., Yamada, K., Suzuki, S., Takase, B., Suzuki, S. and Ishihara, M. (2006) Non-contact respiratory monitoring system using a ceiling-attached microwave antenna. Medical and Biological Engineering and Computing, 44, 835-840. doi:10.1007/s11517-006-0091-8

[7] Suzuki, S., Matsui, T., Kawahara, H. and Gotoh, S. (2009) Development of a noncontact and long-term respiration monitoring system using microwave radar for hibernating black bear. Zoo Biology, 28, 259-270. doi:10.1002/zoo.20229

[8] Suzuki, S., Matsui, T., Imuta, H., Uenoyama, M., Yura, H., Ishihara, M. and Kawakami M. (2008) A novel autonomic activation measurement method for stress monitoring: Non-contact measurement of heart rate variability using a compact microwave radar. Medical and Biological Engineering and Computing, 46, 709-714. doi:10.1007/s11517-007-0298-3

[9] Droitcour, A., Lubecke, V., Lin, J. and Boric-Lubecke, O. (2001) A microwave radio for Doppler radar sensing of vital signs. IEEE MTT-S International Proceedings of Microwave Symposium Digest, Phoenix, 20-25 May 2001, 175-178.

[10] Lin, J.C. (1992) Microwave sensing of physiological movement and volume change: A review. Bioelectromagnetics, 13, 557-565. doi:10.1002/bem.2250130610

[11] Li, C. and Lin, J. (2008) Random body movement cancellation in Doppler radar vital sign detection. IEEE Transactions on Microwave Theory and Techniques, 56, 31433152. doi:10.1109/TMTT.2008.2007139
[12] Goldberger, A.L. (2006) Clinical electrocardiography: A simplified approach. 7th Edition, Mosby, Maryland Heights.

[13] Shin, J.H., Chee, Y.J., Jeong, D.-U. and Park, K.S. (2010) Nonconstrained sleep monitoring system and algorithms using air-mattress with balancing tube method. IEEE Transactions on Information Technology in Biomedicine, 14, 147-156. doi:10.1109/TITB.2009.2034011

[14] Tanaka, S., Matsumoto, Y. and Wakimoto, K. (2002) Unconstrained and non-invasive measurement of heart-beat and respiration periods using a phonocardiographic sensor. Medical and Biological Engineering and Computing, 40, 246-252. doi:10.1007/BF02348132

[15] International Standardization Organization (ISO) (1996) ISO 7250: Basic human body measurements for technological design.

[16] Watanabe, J. and Tochikubo, O. (2003) Relationship between visceral fat accumulation and hypertension in obese men. Clinical and Experimental Hypertension, 25, 199 208. doi:10.1081/CEH-120019152

[17] Li, C., Cummings, J., Lam, J., Graves, E. and Wu, W. (2009) Radar remote monitoring of vital signs. IEEE Microwave Magazine, 10, 47-56. doi:10.1109/MMM.2008.930675

[18] Li, C. and Lin, J. (2010) Recent advances in Doppler radar sensors for pervasive healthcare monitoring. Proceedings of Asia-Pacific Microwave Conference, Yokohama, 7-10 December 2010, 283-290.

[19] Suzuki, S. and Matsui, T. (2012) Chapter 21: Remote sensing for medical and health care applications. In: Escalante-Ramirez, B., Ed., Remote Sensing-Applications, InTech, Rijeka, 479-492.

[20] O'Rourke, M.F., Kelly, R.P. and Avolio, A.P. (1992) The arterial pulse. Lea \& Febiger, Philadelphia.

[21] Segers, P., Rietzschel, E.R., De Buyzere, M.L., Vermeersch, S.J., De Bacquer, D., Van Bortel, L.M., De Backer, G., Gillebert, T.C. and Verdonck P.R. (2007) Noninvasive (input) impedance, pulse wave velocity, and wave reflection in healthy middle-aged men and women. Hypertension, 49, 1248-1255. doi:10.1161/HYPERTENSIONAHA.106.085480 